\title{
Arbolado urbano, calidad del aire y afecciones respiratorias en seis comunas de la provincia de Santiago, Chile
}

\author{
CARLA CRIOLLO C.*, RODRIGO ASSAR C.**, \\ DANTE CÁCERES L.*** y MARGARITA PRÉNDEZ B.*
}

\begin{abstract}
Urban trees, air quality and respiratory diseases in six communes of the province of Santiago, Chile
\end{abstract}

Ozone $\left(\mathrm{O}_{3}\right)$ is a secondary pollutant, often above the norm in some areas of Santiago in the spring-summer period. It is formed in the atmosphere by photochemical reactions, between nitrogen oxides (NOx) and volatile organic compounds (VOCs), which have an important biogenic contribution (BVOCs). In this research the interrelationship between urban trees, their contribution to atmospheric ozone formation and particulate matter, and respiratory diseases in 6 communes in the province of Santiago was analyzed. The choice of the communes considered the number of inhabitants, the existence of stations monitoring air quality, the percentage of green areas and the geographical distribution. The results showed correlation between the type of species of urban trees, ozone, particulate matter and respiratory diseases: asthma and pneumonia. Necessity for replacement of exotic trees species by native species is concluded.

Key words: Urban trees; tropospheric ozone; particulate matter; respiratory diseases.

\section{Resumen}

El ozono $\left(\mathrm{O}_{3}\right)$ es un contaminante secundario, frecuentemente sobre la norma en algunas áreas de Santiago en el periodo primavera-verano. Se forma en la atmósfera por reacciones fotoquímicas, a partir de óxidos de nitrógeno (NOx) y compuestos orgánicos volátiles (COVs), los cuales tienen un aporte biogénico (COVsB) importante. En esta investigación se analizó la interrelación entre el arbolado urbano, su contribución a la formación de ozono y material particulado atmosféricos, y las afecciones respiratorias en 6 comunas de la provincia de Santiago. La elección de las comunas consideró el número de habitantes, la existencia de estaciones de monitoreo de calidad del aire, el porcentaje de áreas verdes y la distribución geográfica. Los resultados muestran que existe relación entre el tipo de especies del arbolado urbano, el ozono, el material particulado y las enfermedades respiratorias: asma y neumonía. Se concluye la necesidad de considerar el remplazo de especies exóticas por especies nativas.

Palabras clave: Arbolado urbano; ozono troposférico; material particulado; afecciones respiratorias.

\section{Introducción}

El ozono $\left(\mathrm{O}_{3}\right)$ urbano es un contaminante secundario que se forma en la tropósfera mediante una reacción fotoquímica entre los óxidos de nitrógeno (NOx) y los compuestos orgánicos volátiles (COVs).

Los COVs se emiten a la tropósfera en grandes cantidades, tanto por fuentes antrópicas como biogénicas ${ }^{1}$ Las fuentes antrópicas incluyen a

\footnotetext{
* Laboratorio de Química de la Atmósfera, Facultad de Ciencias Químicas y Farmacéuticas, Universidad de Chile. Santiago, Chile.

** Instituto de Ciencias Biomédicas ICBM, Facultad de Medicina, Universidad de Chile, Santiago, Chile.

*** Programa de Salud Ambiental, Escuela de Salud Pública, Facultad de Medicina, Universidad de Chile.
} 
los gases de escape de los vehículos motorizados que queman combustible fósil, a la evaporación de combustibles líquidos, solventes y pinturas. La exhalación humana es también un aporte de isopreno, compuesto químico emitido como subproducto metabólico de la síntesis del colesterol. La concentración promedio de isopreno en la respiración está en el rango de $0,065 \pm 0,058 \mathrm{mg} /$ $\mathrm{L}^{2}$. Las fuentes de emisión naturales (biogénicos, COVsB) más importantes son: la vegetación, los océanos, las aguas superficiales continentales, los sedimentos, la descomposición microbiana de material orgánico, los depósitos geológicos de hidrocarburos y los volcanes ${ }^{3}$.

Los COVsB emitidos por las plantas se producen por lo general como un mecanismo de defensa a la temperatura, insectos y plagas $^{4} \mathrm{o}$ como un mecanismo de comunicación entre las plantas $^{5}$. Son compuestos altamente reactivos, cuyos dobles enlaces de carbono-carbono, son fácilmente atacados por radicales libres en la atmósfera; los más importantes son: el isopreno (2-metil-1,3 butadieno, $\mathrm{C}_{5} \mathrm{H}_{8}$, , los monoterpenos (dos unidades de isopreno) de menor volatilidad que isopreno, los sesquiterpenos (tres unidades de isopreno) y algunos compuestos oxigenados como aldehídos y cetonas. Son aromáticos al olfato y de baja solubilidad en agua. El isopreno y los monoterpenos constituyen la fracción más importante de los COVsB y se ha estimado que en promedio se emiten 15 o más monoterpenos por especie vegetal ${ }^{6}$.

Factores ambientales importantes para la emisión son la intensidad de la luz, la humedad relativa y la temperatura y la respuesta es especie específica. Sus mayores concentraciones se encuentran entre las 9:00 am y 5:00 pm del día, horas que corresponden a la mayor radiación solar $^{7}$. Los monoterpenos se suelen acumular en estructuras especiales y liberarse dependiendo de las necesidades, en función de la temperatura de la hoja ${ }^{8}$, la presión de vapor, el área de superficie de interfase entre la hoja y el aire circundante y la humedad del aire. También afectan la emisión la concentración de $\mathrm{CO}_{2}$ atmosférico y la nutrición de la planta ${ }^{9}$.

Históricamente el arbolado urbano ha sido parte del paisaje de las ciudades otorgando beneficios ambientales, sociales y económicos ${ }^{10}$; entre ellos los más comúnmente mencionados son: disminución de la escorrentía de aguas lluvias, mejora de la calidad del aire, disminución de la isla de calor y proporción de sombra ${ }^{11}$ positivo efecto sobre la salud humana ${ }^{12,13}$, aumento de la interacción ciudadana y valorización económica ${ }^{14}$.

En Chile, el arbolado urbano es responsabi- lidad de los municipios de cada comuna, observándose diferencias en la gestión dependiendo del sector económico al cual pertenezca ${ }^{15}$; existe injustica ambiental a nivel comunal debido a la desigualdad socio-económica, siendo las comunas más pobres las menos arborizadas ${ }^{16} \mathrm{y}$ las que presentan mayores niveles de contaminación ${ }^{17}$.

A nivel internacional, los efectos del ozono troposférico en la salud humana se han estudiado durante décadas. Nivel de concentración, período de exposición y sensibilidad individual son los factores más importantes para determinar la magnitud de los efectos. Los estudios epidemiológi$\cos$ de series de tiempo muestran asociaciones positivas débiles entre la mortalidad y el ozono con niveles diarios de $120 \mu \mathrm{g} / \mathrm{m}^{3} \mathrm{~N}$ (calculado como media móvil de $8 \mathrm{~h}$ e independiente de los efectos de material particulado), que es la norma actualmente vigente en Chile. Por ello, el valor máximo recomendado por la Organización Mundial de la Salud en 2011 es de $100 \mu \mathrm{g} / \mathrm{m}^{3} \mathrm{~N}$ como promedio móvil de $8 \mathrm{~h}^{18}$; ese mismo año la OMS señala que los problemas de mayor preocupación son por: el aumento en las admisiones hospitalarias, la exacerbación del asma y las inflamaciones pulmonares. Se ha demostrado la relación entre concentraciones elevadas de ozono y el asma en niños que realizan actividad física ${ }^{19}$; también se ha demostrado el aumento en la tasa de mortalidad a corto plazo, debido a aumentos en las concentraciones de ozono en la atmósfera ${ }^{20}$. A su vez, la $\mathrm{OECD}^{21}$ afirma que el incremento de la contaminación ambiental por ozono podría incrementar en el futuro las muertes prematuras por fallas respiratorias y muertes prematuras de niños (Figura 1).

Con los años, Chile ha experimentado cambios en el tipo de enfermedades que llevan a la muerte de la población del país. En los años 50, las enfermedades respiratorias (neumonías y bronconeumonías) eran padecidas por niños. En los últimos años la información acumulada en

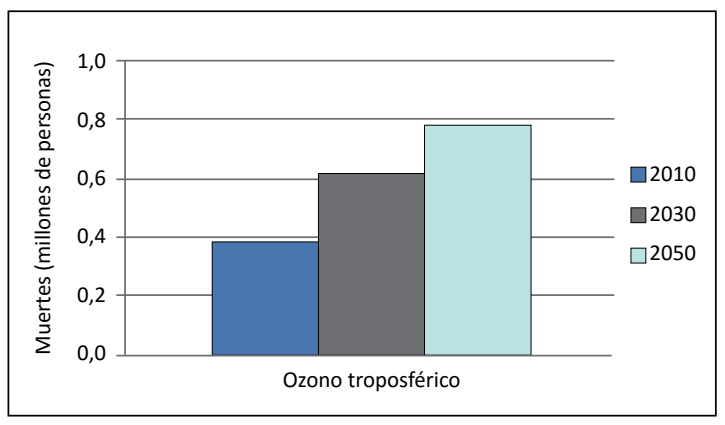

Figura 1. Muertes prematuras globales por riesgos ambientales: Base 2010 al 2050; Fuente: OECD ${ }^{21}$. 

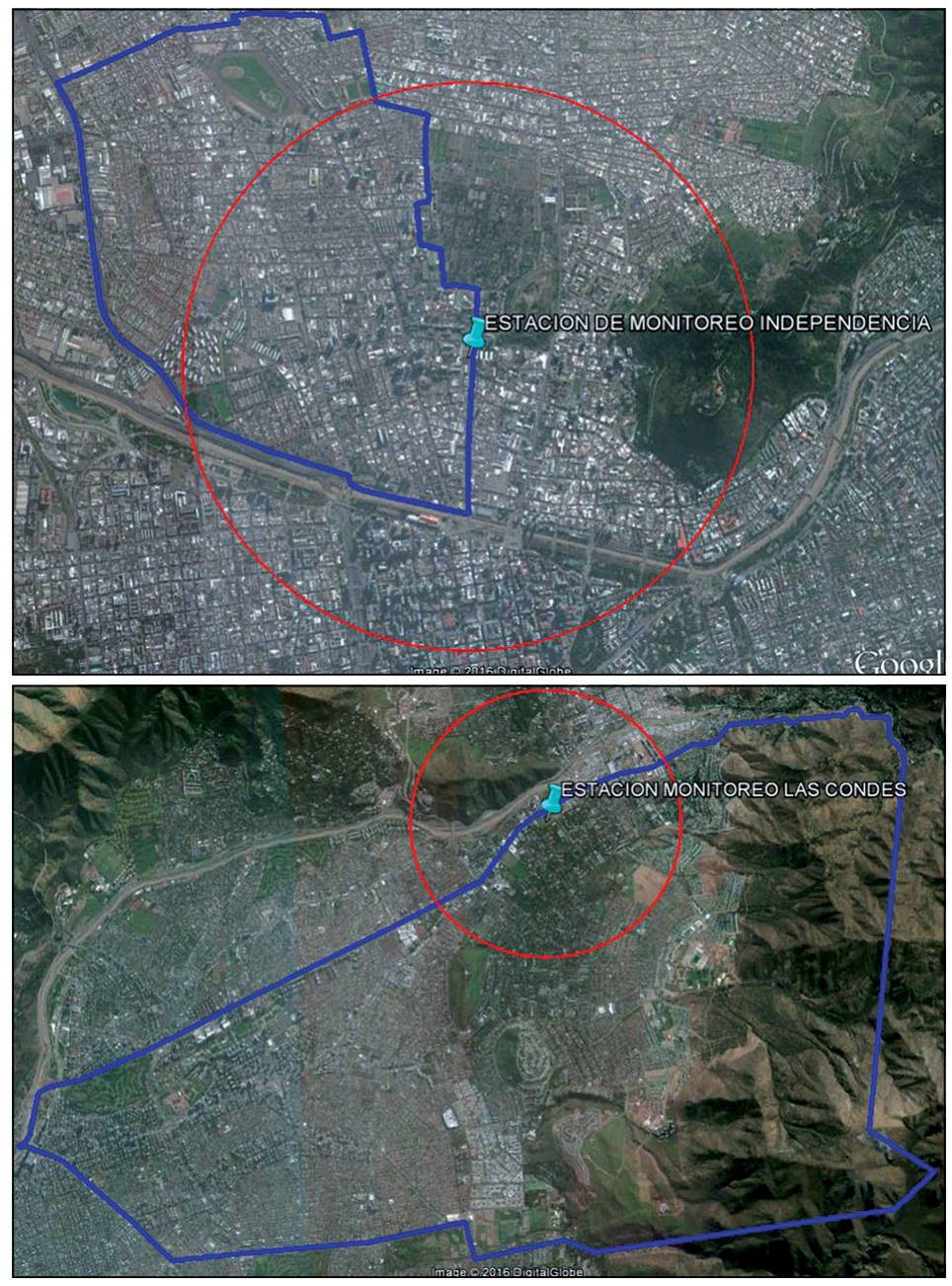

Figura 2. Radio de $2 \mathrm{~km}$ de representatividad de las estaciones de monitoreo para la comunas de Independencia y Las Condes.

DEIS $^{22}$ muestra el aumento en la tasa de muertes por enfermedades respiratorias para los habitantes de la Región Metropolitana (RM) y para el grupo etario sobre los 65 años. Por lo demás las enfermedades respiratorias están dentro de las 10 primeras causas de muerte en Chile $^{23}$ y diferentes investigadores informan de la relación contaminación atmosférica/salud ${ }^{24-29}$.

El objetivo de esta investigación fue estudiar la relación entre la contaminación atmosférica de ozono, el tipo de arbolado urbano público y la salud respiratoria humana para comunas seleccionadas de la provincia de Santiago.

\section{Materiales y Método}

\section{Area de estudio}

Para el inventario del arbolado urbano público se utilizó la información obtenida desde la correspondiente página web de las comunas de la provincia de Santiago, que informan el detalle del número de árboles por especie. Se seleccionaron 6 comunas Maipú, La Florida, Recoleta, Vitacura, La Reina y Las Condes utilizando los siguientes criterios de selección: población comunal, presencia de estaciones de monitoreo de contaminantes del aire (red MACAM), inventario de áreas verdes, porcentaje de áreas verdes por habitante y distribución dentro de la Región Metropolitana. Maipú, La Florida, Santiago y Las Condes tienen el mayor porcentaje de población; Vitacura, La Reina y Las Condes, tienen el mayor porcentaje de áreas verdes; Las Condes, la Florida y Vitacura, cuentan con información sobre estado de salud y edad de los individuos por especie arbórea. Maipú, no cuenta con el detalle de estos parámetros.

Para el cálculo de la población se usaron las proyecciones realizadas para el año 2014 en el censo $2002^{30}$. Para el registro de contaminantes se consideraron las estaciones de monitoreo del 


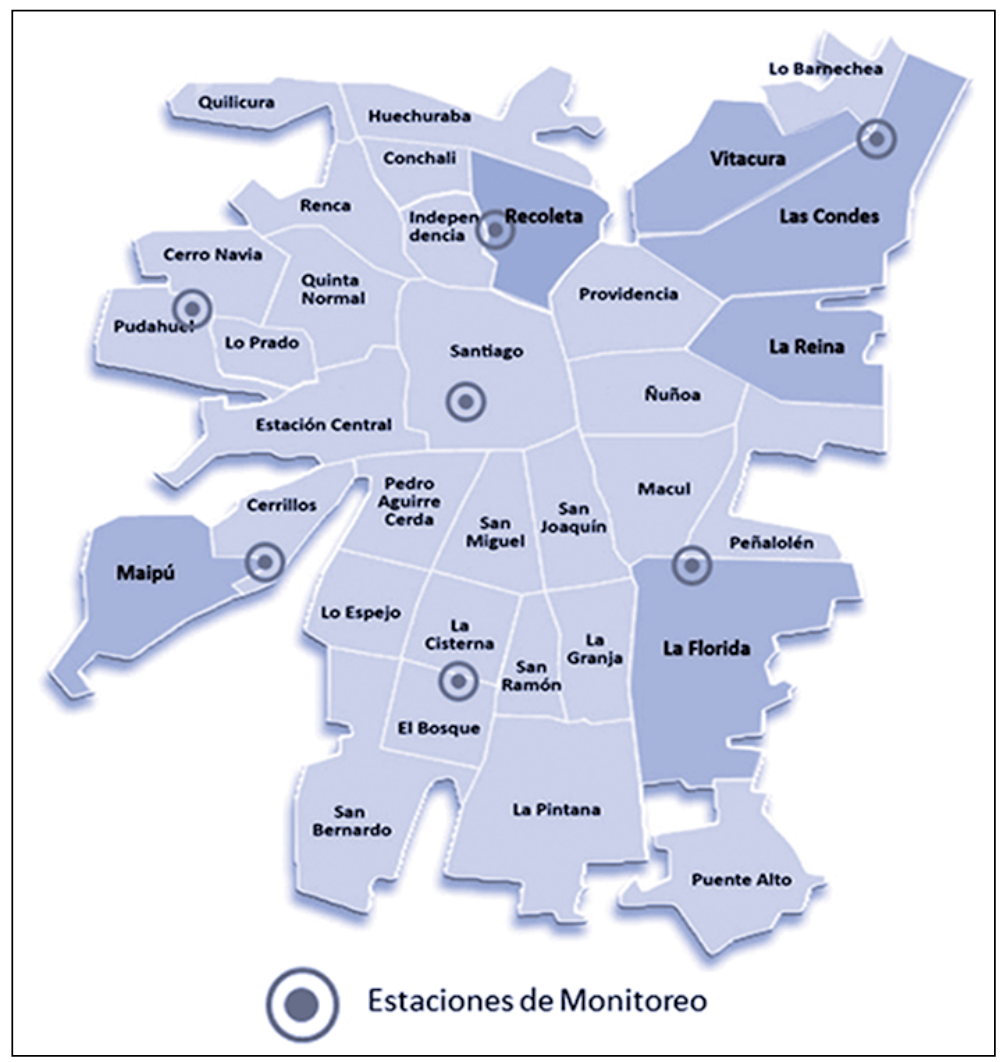

Figura 3. Región Metropolitana con distribución geográfica de las comunas seleccionadas.
SINCA (Sistema de Información Nacional de Calidad del Aire) con un registro completo de material particulado $\mathrm{MP}_{10}$ y $\mathrm{MP}_{2,5}$, y $\mathrm{O}_{3}$, y su representatividad en un radio de $2 \mathrm{~km}$. Así, la estación de Cerrillos incluye parte de la comuna de Maipú, la de Independencia incluye parte de la comuna de Recoleta y la estación de Las Condes, incluye parte de la comuna de La Reina (Figura 2). La Figura 3 muestra la distribución espacial de las comunas seleccionadas.

\section{Egresos hospitalarios}

Se utilizó la información oficial de los egresos hospitalarios del Departamento de Estadísticas e Información de Salud (DEIS) al año 2015 correspondientes a los egresos de personas desde un servicio de salud: Fondo Nacional de Salud (FONASA), Instituciones de Salud Previsional (ISAPRES), Caja de Previsión de la Defensa Nacional (CAPREDENA), Caja de Previsión de Carabineros (DIPRECA), Fuerzas Armadas y sin previsión de salud. Se consideraron 5 años, 2007 a 2011, siendo 2011 el último año disponible en el DEIS al momento de realizar el análisis estadístico. Las causas de los egresos hospitalarios se agruparon según la Clasificación CIE $10^{32}$, implementadas por el Ministerio de Salud de
Chile, MINSAL, a partir del año 1997, ratificada en el año $2008^{33}$. Se seleccionaron los códigos relacionados con las enfermedades respiratorias $\mathrm{J} 12$ a J18, correspondientes a las enfermedades asociadas a Neumonías y J45 y J46, correspondientes a las enfermedades relacionadas al asma y otros estados asmáticos. El análisis incluyó ambos sexos sin distinción de edad ni clasificación de servicio de salud y sólo se consideraron los egresos hospitalarios, que corresponden al retiro del paciente del establecimiento de salud, sin incluir los casos de muerte.

\section{Análisis estadístico}

La extracción y tabulación de los datos se realizó mediante el programa estadístico "Statistical Product and Service Solutions", SPSS. El análisis utilizó el programa de datos -R mediante un análisis de varianza ANOVA. Se buscaron las correlaciones entre las variables de salud y las concentraciones de material particulado $\mathrm{MP}_{2,5} \mathrm{y}$ de ozono. Las concentraciones de $\mathrm{MP}_{2,5}$, corresponden a valores de $24 \mathrm{~h}$ y las concentraciones de ozono al promedio móvil de $8 \mathrm{~h}$.

Se analizó el efecto de la estacionalidad y el efecto del ozono independiente de la estacionalidad (ozono desestacionalizado). La estaciona- 


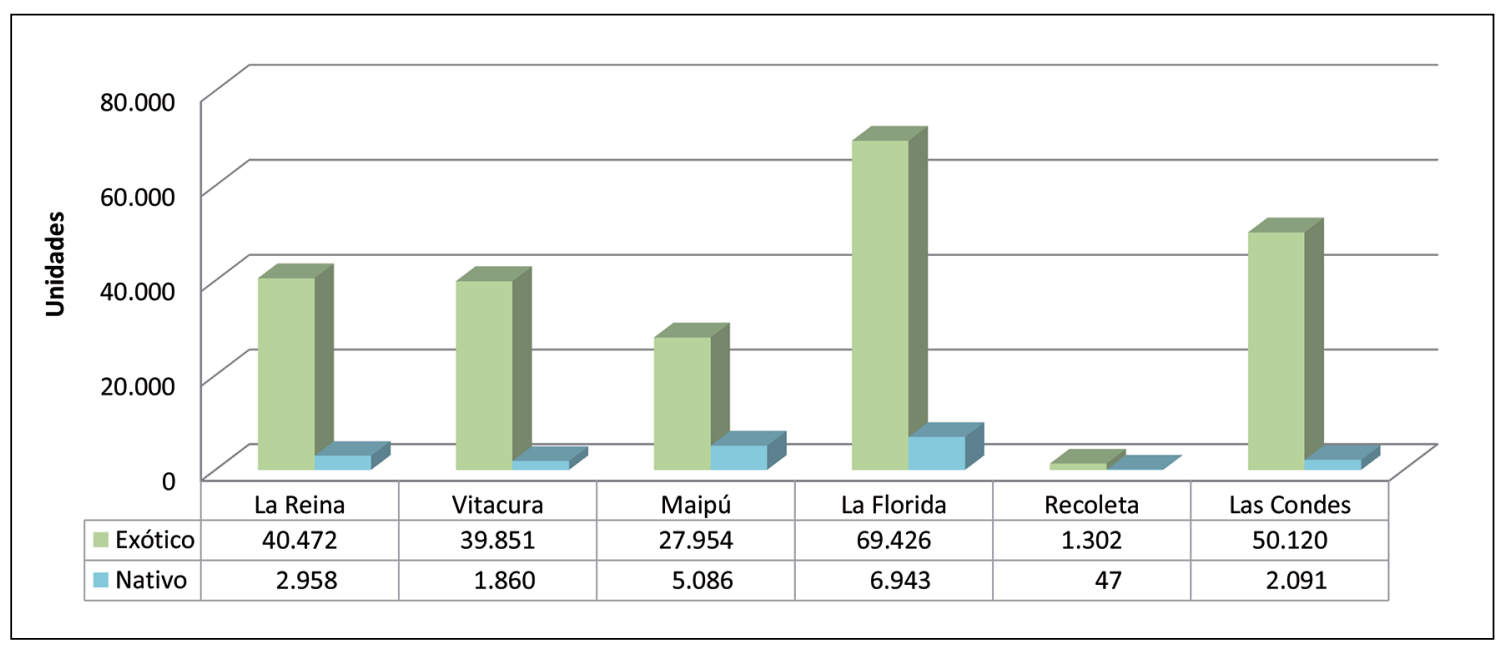

Figura 4. Número de individuos de árboles exóticos y nativos para comunas de La Reina, Vitacura, Maipú, La Florida, Recoleta y Las Condes (Fuente páginas web de las respectivas municipalidades).

lidad corresponde al efecto de los meses del año sobre la respuesta de egresos hospitalarios y la forma en que la afectan las temporadas de primavera, verano, invierno y otoño. El ozono desestacionalizado corresponde al efecto del ozono sobre la respuesta eliminando el factor de las estaciones del año. Para recuperar el efecto oculto del ozono en las estaciones primavera y verano, se separó el efecto propiamente del ozono de aquel asociado a la estacionalidad (variable moderadora) en un modelo lineal generalizado con interacciones. Aplicado dicho procedimiento a cada afección, se analizó el efecto del ozono a nivel general y en cada estación.

\section{Resultados y Discusión}

\section{Arbolado urbano}

La Figura 4 muestra la cantidad de especies exóticas y nativas según el inventario oficial de cada comuna, con números tan dispares como 1.349 (1.302 exóticos y 47 nativos) para la comuna de Recoleta y 76.369 (69.426 exóticos y 6.943 nativos) para la de La Florida con un promedio de $92,8 \%$, de especies exóticas en las seis comunas, siendo Maipú la que tiene más vegetación nativa (sólo 84,6\% de exóticos) y Recoleta la con menos $(96,5 \% \text { de especies exóticas })^{28}$.

Al considerar las 20 especies exóticas más comunes en cada comuna sólo 4 de ellas (Acer negundo, Robinia pseudoacacia, Liquidambar styradflua y Prunus ceracifera var. nigra pisardii) constituyen entre el 50 y el $60 \%$ del arbolado comunal, a excepción de Maipú, donde este porcentaje es del 45,7\% y La Reina que es el 71,7\%.
Esto implica que en todas las comunas estudiadas los árboles exóticos constituyen mayoritariamente el arbolado urbano público y además concentrado en unas pocas especies. Las especies nativas constituyen sólo entre el 3,5\% y el 15,4\%.

La participación de la vegetación en la formación de ozono troposférico está directamente relacionada a sus factores de emisión (FE) de isopreno y monoterpenos, básicamente ${ }^{7}$; estos compuestos reaccionan con diferentes especies químicas en la atmósfera e interfieren el ciclo de los NOx ambientales, formando ozono y algunas otras especies (por ejemplo aerosoles secundarios $\mathrm{MP}_{2,5}$ ) con diferente toxicidad para la salud ambiental y humana. Entre las especies exóticas con altos FE y altos índices potenciales formadores de ozono (IPFO) se encuentran Robinia pseudoacacia, Liquidambar styraciflua, Prunus ceracifera var. nigra pisardii, Prunus ceracifera, Olea europea, Acacia dealbata, Betula pendula. Por el contrario, especies nativas como Quillaja saponaria, Cryptocaria alba, Caesalpinia spinosa, Schinus molle, Acacia caven y Maytenus boaria tienen muy bajos FE e IPFO ${ }^{7,34}$.

Además, muchas especies arbóreas exóticas emiten polen, siendo 9 las predominantes, en emisión de polen de un total de $28^{35}$, como promedio anual; entre ellas, tiene el mayor porcentaje el Platanus orientalis (72\%); le siguen otros como Acer negundo (7,64\%), Fraxinus excelsior $(2,62 \%)$, Olea europaea $(1,46 \%)$, Liquidambar styraciflua (0,31\%), Robinia pseudoacacia $(0,27 \%)$ y Acacia dealbata $(0,11 \%)$. Entre los nativos sólo aparece entre las 28 especies, el Schinus molle $(0,66 \%)$. 
En 5 de las 6 comunas estudiadas (Maipú, Recoleta, Vitacura, La Reina y Las Condes) se encuentran siempre presentes las especies Acer negundo, Robinia pseudoacacia y Liquidambar styraciflua, Fraxinus excelsior y Platanus orientalis. Sólo La Florida no informa presencia de Platanus orientalis, pero además sólo informa 11 especies en su inventario. De este modo, en las 5 comunas indicadas al menos hay un $80 \%$ de especies arbóreas alérgenas en su arbolado urbano pú-

Tabla 1. Nombres científico y común de las especies exóticas y nativas del arbolado urbano de 6 comunas de la provincia de Santiago

\begin{tabular}{|ll|}
\hline Nombre científico & Nombre común \\
Especies exóticas & \\
Acer negundo & Acer \\
Acacia dealbata & Aromo del país \\
Betula pendula & Abedul \\
Brachichyton populneus & Brachichiton \\
Fraxinus excelsior & Fresno \\
Liquidambar styradflua & Liquidambar \\
Olea europea & Olivo \\
Platanus orientalis & Plátano oriental \\
Prunus ceracifera & Ciruelo verde \\
Prunus ceracifera var. Nigra pisardii & Ciruelo rojo \\
Quercus suber & Corcho \\
Robinia pseudoacacia & Acacio \\
Especies nativas & \\
Acacia caven & Espino \\
Caesalpinia spinosa & Tara \\
Cryptocaria alba & Peumo \\
Maytenus boaria & Maitén \\
Quillaja saponaria & Quillay \\
Schinus molle & Pimiento \\
\hline
\end{tabular}

blico $^{28}$. La Florida registra un $10,8 \%$ de especies alergenas. Para el área metropolitana de Santiago los niveles de protección de salud recomendados por la Academia Americana de Alergia, Asma e Inmunología, indican que el $25,5 \%$ de los días en un año promedio se encuentran en alta o muy alta concentración de alergenos provenientes de árboles, pastos y hierbas, y el 14\% de los días del año (51 días) con una exposición a niveles altos y muy altos de alergenos, entre los meses de agosto y octubre, atribuibles sólo a árboles, siendo el mes de septiembre particularmente intenso ${ }^{35}$.

En la Tabla 1 se presenta un glosario que incluye los nombres científicos y comunes de las especies exóticas y nativas mencionadas en este artículo.

\section{Análisis estadístico de casos de asma por comuna}

La Figura 5 muestra las relaciones estadísticas, con significancia del $95 \%$ entre estacionalidad, ozono, $\mathrm{MP}_{2,5}$ y ozono desestacionalizado para las comunas estudiadas.

En ninguna de las comunas estudiadas el $\mathrm{MP}_{2,5}$ es significativo para los egresos hospitalarios por asma. En las comunas de Vitacura, Maipú, La Florida y Recoleta, las concentraciones de ozono no se asocian estadísticamente con la cantidad de egresos hospitalarios. Sin embargo, el ozono desestacionalizado fue significativo estadísticamente (flecha arriba), es decir que las concentraciones de ozono desestacionalizado influyen directamente en la respuesta de egresos hospitalarios.

En La Reina la concentración de ozono presenta un comportamiento estadísticamente significativo con la estacionalidad de forma inversa (negativo) y con el ozono desestacionalizado, de forma directa (positivo), lo que significa que la presencia de ozono siempre produce un efecto,

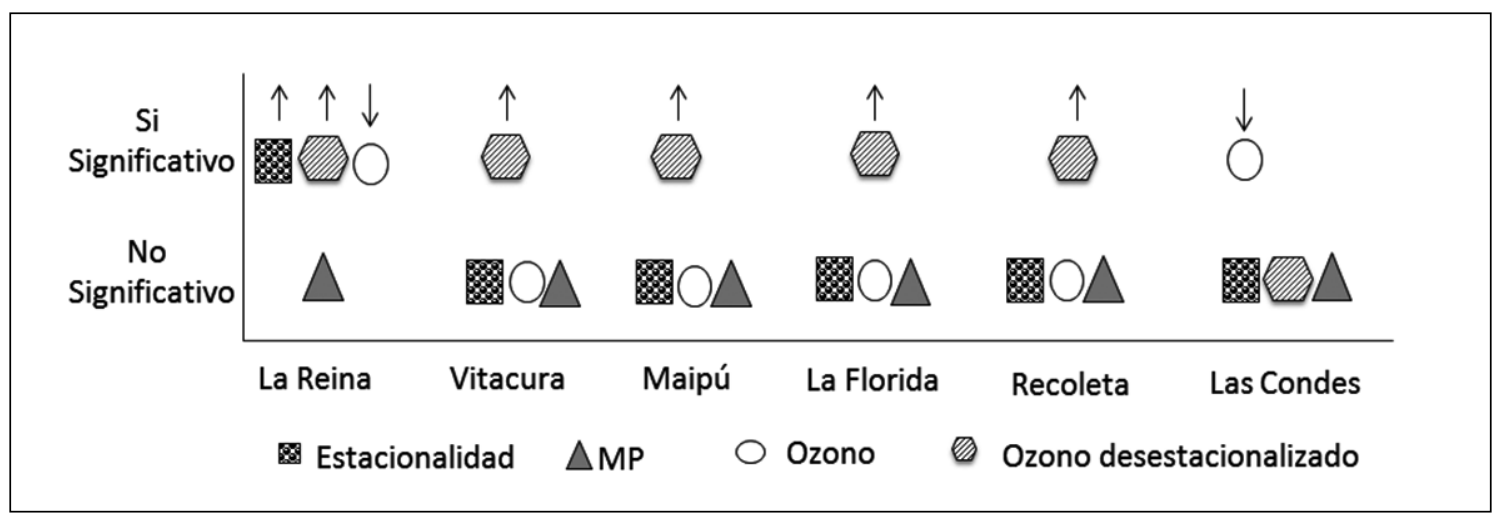

Figura 5. Relación de estacionalidad, material particulado, ozono y ozono desestacionalizado, para casos de asma, en las comunas de La Reina, Vitacura, Maipú, La Florida, Recoleta y Las Condes. 
en cambio, el material particulado no fue significativo en esta comuna.

El asma es una patología de características inflamatoria y bronco-obstructiva, por lo que puede relacionarse con la presencia de ciertos contaminantes ambientales, ya que se podría producir inflamación por partículas de pequeño tamaño ingresando al organismo. Para una mejor explicación del comportamiento que muestra esta comuna sería necesario ahondar más en otros aspectos, como por ejemplo posibles sinergias entre todos los factores estudiados $\mathrm{u}$ otros no considerados aquí. En La Reina el asma está significativamente vinculada a la estacionalidad. De algún modo, no claro aún, el ozono es un compuesto estadísticamente vinculado a la afección respiratoria de asma durante todo el año, sobre los habitantes de la comuna de La Reina. Para esta comuna se utilizaron las concentraciones de contaminantes entregadas por la estación de monitoreo de Las Condes, lo cual podría no representar cabalmente la realidad. Dada la información planteada, sería aconsejable que esta comuna tuviera su propia estación de monitoreo de, al menos, ozono, NOx y COV.

En la comuna de Las Condes, las variables de estacionalidad, $\mathrm{MP}_{2,5}$ y ozono desestacionalizado no son estadísticamente significativas. El ozono estacional es significativo y negativo, al igual que en La Reina. Las Condes informa un 95\% de árboles exóticos en su arbolado urbano, de los cuales el 15\% corresponde a Acer negundo y el $5,4 \%$ a platanus orientalis. Estas dos especies dan cuenta del $80 \%$ de las especies alergenas de la comuna, con una emisión estacional.

\section{Análisis estadístico de casos de neumonía}

La Figura 6 muestra las correlaciones estadísticamente significativas al $95 \%$ con la estacionali- dad, el ozono, el $\mathrm{MP}_{2,5}$ y el ozono desestacionalizado para las seis comunas estudiadas, mostrando que la estacionalidad influye de forma directa en la respuesta de egresos hospitalarios y las concentraciones de ozono influyen inversamente sobre los egresos hospitalarios por neumonías. Las concentraciones de $\mathrm{MP}_{2,5}$ y las del ozono desestacionalizado están estadística y directamente vinculadas a los egresos hospitalarios por neumonías para todas las comunas a excepción de Las Condes. En la comuna de Las Condes, el $\mathrm{MP}_{2,5}$ y el ozono desestacionalizado no son significativos. En Las Condes las concentraciones de $\mathrm{MP}_{2,5}$ son las más bajas registradas en la ciudad de Santiago, en cambio, las concentraciones de ozono suelen ser las más altas. Por otra parte, esta comuna junto con La Reina, tienen los niveles socio-económicos más altos del grupo de comunas analizadas.

El comportamiento observado se podría intentar explicar desde la etiología de la afección. La neumonía es una enfermedad generada por un agente patógeno infeccioso, por lo que la etiología de esta enfermedad es bastante compleja, ya que depende de variados factores como lo son la presencia del patógeno, niveles del patógeno, vulnerabilidad del huésped en términos de inmunosupresión y grado de agresividad del microorganismo, que a su vez puede depender de muchos otros factores, entre ellos, las condiciones ambientales. De esta forma, la respuesta de los egresos hospitalarios de aumentar cuando disminuye la concentración de ozono o disminuir cuando aumenta la concentración de ozono, podría explicarse mejor con una desagregación de los datos como sería un análisis más profundo de las etiologías de cada una de las afecciones para los códigos CIE-10 analizando grupo etario de los egresos hospitalarios y niveles de significancia.

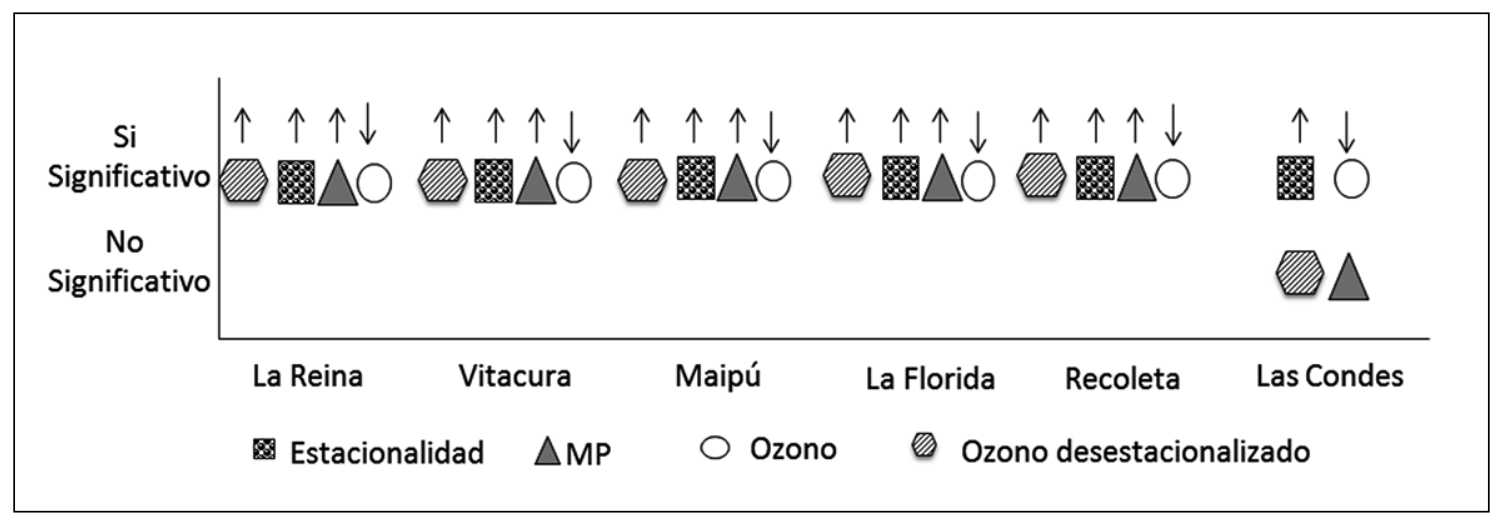

Figura 6. Comunas y relación de estacionalidad, material particulado, ozono y ozono desestacionalizado, para casos de neumonía, en las comunas de La Reina, Vitacura, Maipú, La Florida, Recoleta y Las Condes. 
Tabla 2. Ubicaciones geográficas de algunos hospitales de la provincia de Santiago.

\begin{tabular}{|lll|}
\hline Hospital /Instituto & Comuna & Dirección \\
\hline Hospital San José & Independencia & San José 1136 \\
\hline Hospital Clínico de Niños Roberto del Río & Independencia & Avda. Profesor Zañartu 1085 \\
\hline Hospital San Juan de Dios de Santiago & Santiago & Huérfanos 3255 \\
\hline Hospital Félix Bulnes Cerda & Quinta Normal & Leoncio Fernández 2655 \\
Hospital Clínico San Borja-Arriarán & Santiago & Santa Rosa 1234 \\
Hospital del Salvador & Providencia & Avda. Salvador 364 \\
Hospital de Niños Dr. Luis Calvo Mackenna & Providencia & Antonio Varas 360 \\
\hline Instituto Nacional de Enfermedades Respiratorias y Cirugía del Tórax & Providencia & José M. Infante 717 \\
\hline Instituto Nacional de Neurocirugía & Providencia & José M. Infante 553 \\
\hline
\end{tabular}

De los análisis anteriormente revisados para asma y neumonía se puede apreciar la relación entre egresos hospitalarios, ozono y material particulado. Esto hace notar la importancia de la calidad del aire para las personas en general, y en especial, para la población con salud deprimida, como es el caso de la población que asiste a centros de salud y hospitales, por lo que el emplazamiento de éstos debería estar alejado de las mayores fuentes de contaminación como son los espacios de alto flujo vehicular, por ejemplo, en especial respecto de la neumonía. Muchas de las personas que acuden a un centro de salud pueden presentar esta afección respiratoria la que puede verse acrecentada en situaciones de contaminación atmosférica; a su vez, ellas pueden contagiar a otras, que frente a la contaminación, pueden ser más susceptibles al contagio. Las características medioambientales en el hogar tendrían una relación positiva entre la incidencia de neumonía adquirida y los episodios agudos de contaminación del aire. Otro factor puede ser el tabaco, al interior del hogar ${ }^{36}$.

La Tabla 2 muestra la proximidad de algunos hospitales de Santiago a calles de alto flujo vehicular, especialmente los hospitales San José, Roberto del Rio y San Borja Arriarán. Se ha encontrado correlación directa mayor al 95\% entre el flujo vehicular y la presencia de material particulado antropogénico depositado en las calles. La correlación es mayor al 99,9\% en calles y hojas de Platanus orientalis en la comuna de Vitacura $^{37}$, la cual en nuestro caso muestra una correlación directa entre $\mathrm{MP}_{2,5}$ (gran aporte vehicular) y ozono (contaminante secundario) desestacionalizado.

Otro efecto interesante de analizar en salud, es la presencia simultánea de contaminantes atmosféricos y su interacción entre sí. La mayoría de los estudios suponen un efecto aditivo entre los contaminantes, de tal manera que el efecto es igual a la suma de las estimaciones de los efectos de los contaminantes individuales. Esto puede no ser así, debido a que los contaminantes también pueden tener efecto sinérgico, antagónico o combinado, por lo que el efecto en salud no corresponde siempre a un efecto aditivo ${ }^{38}$. En Santiago, se encontraron también correlaciones entre $\mathrm{CO}$, $\mathrm{NO}_{2}$ y material particulado y las admisiones hospitalarias en el período 2004-2007, pero no con ozono $^{39}$. Sería importante evaluar los diferentes factores que afectan la contaminación atmosférica y la exposición a contaminantes múltiples en el aire sobre la población dada la deteriorada calidad del aire de la ciudad de Santiago.

\section{Conclusiones}

Enfermedades de características inflamatorias e infecciosas del sistema respiratorio como lo son asma y neumonía, presentan relación para las seis comunas estudiadas de la provincia de Santiago con los precursores de ozono, provenientes del arbolado urbano presente en ellas.

El remplazo de ciertas especies exóticas por otras nativas contribuiría a disminuir las emisiones de los compuestos orgánicos volátiles y su potencial formador de ozono y de polen, contribuyendo a mejorar la calidad del aire a nivel comunal y de la salud de la población.

La mayor desagregación de los datos de salud con un estudio profundo de la etiología de la enfermedad por código específico de afección, sería el paso a seguir para lograr un mayor detalle de los efectos en salud que la contaminación atmosférica y la presencia de ozono en la tropósfera pueden estar generando en la población. 


\section{Agradecimientos}

Los autores agradecen al proyecto REDES 140176 de Conicyt por el financiamiento a esta investigación.

\section{Referencias}

1.- ROUKOS J, RIFFAULT V, LOCOGE N, PLAISANCE $\mathrm{H}$. VOC in an urban and industrial harbor on the french North Sea coast during two contrasted meteorological situations. Environ Pollut 2009; 157: 3001-9.

2.- KINOYAMA M, NITTA H, WATANABE A, UEDA H. Acetone and isoprene concentrations in exhaled breath in healthy subjects. J Health Sci 2008; 54:471-7.

3.- FEHSENFELD F, CALVERT J, FALL R, GOLDAN P, GUENTHER A, HEWITT C, et al. Emissions of volatile organic compounds from vegetation and the implications for atmospheric chemistry. Global biogeochemistry 1992; 6: 389-430.

4.- KANSAL A. Sources and reactivity of NMHCs and VOCs in the atmosphere: a review. J Hazard Mater 2009; 166: 17-26.

5.- PEÑUELAS J, STAUDT M. BVOCs and global change. Trends Plant Sci 2010; 15: 133-44.

6.- ARNETH A, MONSON R K, SCHURGERS G, NIINEMETS U, PALMER P I. Why are estimates of global terrestrial isoprene emissions so similar and why is this not so for monoterpenes, Atmospheric Chemistry and Physics 2008; 8: 4605-20.

7.- PRÉNDEZ M, CARVAJAL V, CORADA C, MORALES J, ALARCÓN F, PERALTA H. Biogenic volatile organic compounds from the urban forest of the Metropolitan Region, Chile. Environ Pollut 2013; 183 : 143-50.

8.- FUENTES J, LERDAU M, ATKINSON R, BALDOCCHI D, BOTTENHEIM J, CICCIOLI P, et al. Biogenic hydrocarbons in the atmospheric boundary layer: a review, Bulletin of the American Meteorological Society. 2000; 81: 1537-75.

9.- LORETO F, SCHNITZLER P. Abiotic stresses and induced BVOCs, Trends Plant Sci 2010; 15: 154-66.

10.- MULLANEY J, LUCKE T, TRUEMAN S. A review of benefits and challenges in growing street trees in paved urban environments. Landscape and Urban Planning 2015; 134: 157-66.

11.- PATAKI D E, CARREIRO M M, CHERRIER J, GRULKE N E, JENNINGS V, PINCETL S, et al. Coupling biogeochemical cycles in urban environments: ecosystem services, green solutions, and misconceptions. Review. Frontiers in Ecology and the Environment 2011; 9: 27-36, doi: 10.1890/090220.

12.- LOVASI G S, QUINN J W K, NECKERMAN M, PERZANOWSKI M S, RUNDLE A. Children Living in Areas with More Street Trees Have Lower Prevalence of Asthma. J Epidemiol Community Health 2008; 62: 647-49.

13.- DONOVAN G H, BUTRY D T, MICHAEL Y L, PRESTEMON J P, LIEBHOLD A M, GATZIOLIS D, et al. The Relationship Between Trees and Human Health. Evidence from the Spread of the Emerald Ash Borer. Am J Prev Med 2013; 44: 139-45.

14.- ESCOBEDO F J, KROEGER T, WAGNER J E. Urban forests and pollution mitigation: Analyzing ecosystem services and disservices. Environ Pollut 2011; 159: 2078-87.

15.- HERNÁNDEZ J. La situación del arbolado urbano en Santiago. Revista de Urbanismo 2008; 18 (junio) DOI: 10.5354/0717-5051.2008.272 Disponible en: http:// www.revistaurbanismo.uchile.cl/index.php/RU/article/ viewFile/272/218. Acceso: 10.08.2016.

16.- MMA, Ministerio del medio ambiente. Áreas verdes e ingreso per cápita de las comunas de la provincia de Santiago. Informe estado del medioambiente 2011, Capítulo 6. (en línea <http://www.mma.gob.cl/1304/ w3-article-52016.html, acceso: 09 de junio 2016).

17.- SARRICOLEA P, ROMERO H. (2008). Justicia ambiental del área metropolitana de Santiago (ams) debido a la configuración de las islas de calor urbano y la contaminación por material particulado. XXIX Congreso Nacional y XIV Internacional de Geografía. 14-17 de octubre, Universidad de la Frontera, Temuco, Chile.

18.- OMS. 2014. Calidad del aire (exterior) y salud. Nota descriptiva No313, Centro de Prensa. (En línea]. <http:// www.who.int/mediacentre/factsheets/fs313/es/ acceso: 07 de julio 2016).

19.- MCCONNELL R, BERHANE K, GILLILAND F, LONDON S, ISLAM T, GAUDERMAN J, et al. Asthma in exercising children exposed to ozone: a cohort study. Lancet 2002; 359: 386-91.

20.- BELL M, MCDERMOTT A, ZEGER S, SAMET J, DOMINICI F. Ozone and Short-term Mortality in 95 US Urban Communities, 1987-2000. 2004; 292: 2372-8.

21.- OECD Organization for Economic Co-operation and Development. Environmental Outlook to 2050: The Consequences of Inaction. OECD Publishing, Paris, Francia (2012).

22.- DEIS, Departamento de Estadísticas e Información de Salud, Ministerio de Salud de Chile. Principales causas de muerte según región, sexo y año de defunción en adultos de 65 y más. Chile, 1997 a 2011. (En línea: http://www.deis.cl/?p=2543, acceso: 23 de mayo de 2015).

23.- DEIS, Departamento de Estadísticas e Información de Salud, Ministerio de Salud de Chile. Primeras causas de defunción en Ambos Sexos, Chile 2000-2010. 2001-2007. (En línea: http://www.deis.cl/wp-content/ uploads/2012/10/10-primeras-causas-de-muerte-Chile-2000-2010.xlsx, acceso: 23 de mayo de 2015).

24.- MATUS P, LUCERO R. Norma Primaria de Calidad del Aire. Rev Chil Enferm Respir 2002; 18: 112-22. 
25.- CAKMAK S, DALES R E, VIDA C B. Components of particulate air pollution and mortality in Chile. Int $\mathrm{J}$ Occup Environ Health 2009; 15: 152-8.

26.- OYARZÚN M, DUSSAUBAT N D, MILLER, M E, LABRA S. GONZÁLEZ S. Efectos proinflamatorios de la contaminación atmosférica. Rev Chil Enferm Respir 2011; 27: 183-90.

27.- LEIVA M, SANTIBÁÑEZ D, IBARRA S, MATUS P, SEGUEL R. A five-year study of particulate matter (PM2.5) and cerebrovascular diseases. Environ Pollut 2013; 181: 1-6.

28.- CRIOLLO C. Análisis de propuestas de contribución del arbolado urbano para la mejora de la calidad del aire en áreas de la provincia de Santiago. Tesis para optar al grado de Magíster en Gestión y Planificación Ambiental. Programa Interfacultades, Universidad de Chile 2015. http://mgpa.forestaluchile.cl/ tesis.html.

29.- RUIZ-RUDOLPH P, ARIAS N, PARDO S, MEYER M, MESÍAS S, GALLEGUILLOS C, et al. Impact of large industrial emission sources on mortality and morbidity in Chile: A small-areas study. Environ Int 2016; 92-93: 130-8.

30.- INE. Instituto Nacional de Estadísticas. Estadísticas demográficas y vitales. Comunas. Evolución de algunos indicadores demográficos 2001-2020. (En línea. <http:// www.ine.cl/canales/chile_estadistico/familias/demograficas_vitales.php acceso: 09 de junio 2016).

31.- DEIS, Departamento de Estadísticas e Información de Salud, Ministerio de Salud de Chile. Menú egresos hospitalarios (en línea: http://intradeis.minsal.cl/egresoshospitalarios/menu_publica_nueva/menu_publica_nueva.htm, acceso: 23 de mayo de 2015).

32.- DEIS, Departamento de Estadísticas e Información de Salud, Ministerio de Salud de Chile. CIE-10 (En línea:
http://ais.paho.org/classifications/Chapters/index.htm, acceso: 23 de mayo de 2015).

33.- Decreto $N^{\circ} 1-08$. Norma_Revisión_Estadística internacional de enfermedades y problemas relacionados con la salud para la codificación de las causas de muerte y de enfermedad. Subsecretaria de Salud pública. Ministerio de salud, Santiago, Chile (En línea: http://juridico1. minsal.cl Acceso: 30 mayo 2015).

34.- PRÉNDEZ M, CORADA K, MORALES J. Natural organic compounds from the urban forest of the Metropolitan Region, Chile: impact on air quality (Chapter 5) In: Volatile Organic Compounds. Editor Khaled Chetehouna. 2014 Nova Sciences Publishers Inc. Pages 103-42. New York, USA (2014).

35.- TORO R, CÓRDOVA A, CANALES M, MORALES R, MARDONES P, LEIVA M. Trends and threshold exceedances analysis of airborne pollen concentrations in Metropolitan Santiago Chile. PLoS ONE 2015; 10(5): e0123077. doi:10.1371/journal.pone.0123077

36.- VALDIVIA G. Epidemiología de la neumonía del adulto adquirida en la comunidad. Rev Chil Enferm Respir 2005; 21: 73-80.

37.- MUÑOZ D. Estimación del material particulado y su composición elemental sobre superficies urbanas mediante técnicas fisicas no destructivas. Tesis de Magister en Química, Facultad de Ciencias Químicas y Farmacéuticas, Universidad de Chile, (2016). Disponible en www.tesis.uchile.cl/

38.- OAKES M, BAXTER L, LONG TC. Evaluating the application of multipollutant exposure metrics in air pollution health studies. Environ Int 2014; 69: 90-99.

39.- ULRICH F, LEITTE A M, SUPPAN P. Multiple exposures to airborne pollutants and hospital admissions due to diseases of the circulatory system in Santiago de Chile. Sci Total Environ 2014; 468-9: 746-56.

Correspondencia a:

Dra. Margarita Préndez B.

Laboratorio de Química de la Atmósfera

Facultad de Ciencias Químicas y Farmacéuticas, ,

Universidad de Chile,

Sergio Livingstone 1007, Santiago, Chile.

mprendez@ciq.uchile.cl 